\title{
A molecular dynamics simulation study of semi-solid-state Fe: high temperature elasticity and void formation in liquid
}

\author{
B. Monasse ${ }^{1}$, Ch. Pradille ${ }^{1}$ and Y. Chastel ${ }^{1,2}$ \\ 1 Mines-Paristech, Centre de Mise en Forme des Matériaux, BP 207, 06904 Sophia-Antipolis, France \\ e-mail: bernard.monasse@mines-paristech.fr \\ 2 Present address: Renault DIMat, 78288 Guyancourt, France
}

\section{Key words:}

Molecular dynamics; elasticity; cavitation; iron; crystallization

\begin{abstract}
Hot tearing defects appear during the last steps of casting solidification of dendrites. The crystalline structure traps a small amount of liquid metal, which is depressed, and cavities are spontaneously initiated. Molecular dynamics (MD) simulations of pure iron are able to predict, on one hand, the high temperature elastic modulus of crystals, and on the other hand, the cavitation event in liquid metal and near the solid-liquid growth front. High values of elastic tensor components are predicted close to the melting temperature. We conclude that a weakening of the mechanical properties of the crystal is not the reason for failure initiation. The crystals are thinned by partial melting as the effect of negative pressure increases. The cavitation is spontaneously initiated under a very high negative pressure. The cavity expands very quickly and applies a pressure wave to the thinned crystals. We propose that this crystal thinning/pressure wave coupling is able to initiate cracks inside the crystal.
\end{abstract}

Mots-clés :

Dynamique moléculaire ; élasticité ; cavitation ; fer ; cristallisation
Received 30 December 2013 Accepted 26 June 2014
Résumé - La fissuration à chaud des aciers apparaît durant les derniers stades de la solidification des structures dendritiques. La structure cristalline est largement développée et piège une faible quantité de métal fondu qui est mise en dépression et cavite. La dynamique moléculaire (DM) de fer pur permet de prévoir, d'une part, l'évolution du module élastique du cristal à très haute température et, d'autre part, les conditions de cavitation du métal à l'état fondu et à proximité du cristal. Une valeur très élevée du module élastique est prédite même à la température de fusion. Ce n'est certainement pas la cause de l'amorçage de la fissuration. Elle est recherchée dans le phénomène de cavitation. Les cristaux fondent partiellement par l'application d'une dépression. Pour une très forte pression négative la cavitation est amorcée. Le volume de la cavité croit très rapidement vers des cristaux amincis et applique une onde de pression. Nous proposons que ce couplage amincissement/onde de pression peut amorcer la fissuration.

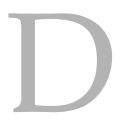
uring the last steps of dendrite development in iron alloys, cavities can initiate and develop to create voids and then cracks [1]. The density difference between the crystal and liquid phases is able to create a pressure depression inside the melt. This effect is enhanced by a confinement of the still liquid phase embedded by crystals at the end of dendrite development. A pressure gradient appears if the melt cavity is connected to the unconfined melt. The pressure gradient must induce a liquid flow through the dendrite array in the mushy zone to feed the zone under low pressure.
The pressure should be unbalanced when the cavity is closed by all the surrounding crystals and initiates cavitation. Crack formation, known as the hot tearing process, should result from these cavitation processes but is still under analysis.

This description follows the hot tearing model proposed by Rappaz et al. [2]. This widely accepted model predicts the fluid velocity to feed these zones by application of Darcy's law applied with a temperature gradient. This model includes the role of alloy composition to predict the hot tearing sensitivity and was successfully compared with 
experimental data in $\mathrm{Al}-\mathrm{Cu}$ alloys [3] and ring-mold solidification experiments [4]. It is able to predict the sharp peak for hot tearing probability as a function of composition with a maximum close to the maximum of the solidification interval $[1,2]$. The three main assumptions of this model for the appearance (initiation) of the first hot tear are the coherency of the dendritic structure in a network, the role of a large negative pressure to initiate void formation and the viscosity effect for feeding the mushy room. The coherency is assumed to be described by uniaxial tensile deformation and not hydrostatic depression [1,2]. The value of the critical pressure to initiate cavitation has a major effect. A low cavitation depression of $-2 \mathrm{kPa}$ was proposed by Rappaz et al. as a key value of their model [1]. Vernede et al. [5] using a granular hot tearing model propose a lower pressure from $-100 \mathrm{kPa}$ to $-500 \mathrm{kPa}$. This value is questioned by a molecular dynamics simulation which results in a higher critical pressure of $-670 \mathrm{MPa}$ for cavitation [6], closer to theoretical values from nucleation in the order of GPa. We apply a similar molecular dynamics simulation for the analysis of melt of iron, while they studied aluminum. The comparison of some results will be useful and we will point out during the text the new advances in this paper. The viscosity depends on the temperature and is known under high hydrostatic compression but is poorly known under depression $[7,8]$.

Molecular dynamics simulations are able to predict behavior and properties in a high variety of conditions. Thus, these simulations can only be done on domains of small dimensions, less than hundreds or thousands of cubic nanometers. These dimensions are very small compared with the domain where the complete phenomenon of hot tearing appears. The simulations are selected to study the elementary processes involved in hot tearing. Four selected processes will be examined by molecular dynamics simulations. An overall proposal mechanism will be proposed from these selected simulations. First, the components of elastic tensor will be predicted for a fcc iron crystal in a large temperature-domain. The objective is to define the mechanical properties at high temperature to find a possible origin of weakness of dendrite organization. The second objective is to predict the critical pressure condition in the iron melt to initiate the cavitation at different undercooling temperatures. The effect of temperature and pressure on viscosity will be defined. This evolution is useful to define the effect of viscosity during the feeding of the mushy zone. Thirdly, the proximity of a crystalline growth front to favor cavitation in the melt will be questioned. The results obtained at the same temperatures on cavitation in the melt and in the semi-solid domain will be compared. The last objective is to analyze the effect of negative pressure and cavitation on the crystalline growth rate.

\section{Simulation procedures}

All the simulations are done with the interatomic potential force-field of pure iron developed by Rosato [9] with the finely optimized parameters checked by Kojima for the fcc phase ( $\gamma$ phase) [10]. This potential model describes the many-body effect with a second moment approximation for tight binding [11]. This force field is chosen for its ability to predict the stability of the fcc crystalline phase and its main properties: the melting temperature $(1710 \mathrm{~K})$, heat of fusion, heat capacity and thermal expansion of the crystalline phase. The $\delta$ phase (bcc), hightemperature stable crystalline phase, is not well predicted by this force-field. This fact explains our choice of the fcc for the simulations of the crystalline phase. All the MD simulations are done with the parallel code DL_POLY [12] with a time step of 3 fs. No dimension of the system is shorter than two times the cut-off of the force-field $(2 \mathrm{~nm})$. The stress components (normal and tangential to each face of the box) are delivered by DL_POLY software, as well as energy individual components, temperature and mean pressure.

A NVT ensemble is applied for fcc crystal, bulk liquid and semi-solid simulations at different temperatures on boxes with periodic conditions. A Nosé-Hoover thermostat is applied with a relaxation time of $10 \mathrm{fs}$. For each simulation, the geometry of the box is adjusted by a trial-and-error procedure to reduce each component of the stress to 
$0 \pm 10$ bars. This relaxed geometry is used to predict the dilatation coefficient of the crystal and melt phases.

The size of the system depends on simulation. 9600 or 10000 atoms are, respectively, necessary to predict the bulk melt and the elastic behavior, while 96000 atoms are applied in an anisotropic-sized box for semisolid simulations. The larger dimension extends along the normal of the crystal/melt interface with the [111] crystalline orientation, i.e. a dense plane. The change of one to three components of a box size for the crystal is able to deduce the components of stress tensors.

The crystal including 10000 atoms is oriented inside the cubic main directions of the simulation box collinear to simple crystallographic axes. All the mechanical properties are measured in the range $300 \mathrm{~K}-1800 \mathrm{~K}$ with a $100 \mathrm{~K}$ temperature step. Poisson's ratio is measured from $0.1 \%, 0.2 \%$ and $0.5 \%$ uniaxial tensile test simulations. Then, the lateral dimensions of the crystal are modified in order to reduce the lateral components of the stress to zero. The elastic modulus is finally calculated along the uniaxial direction for tension from these plane stress simulations, as a function of temperature. The three components C11, C12 and C44 of the elastic tensor of fcc crystal are predicted as a function of temperature. For each temperature the unstressed crystal is submitted to a series of deformations along different pathways with a $0.05 \%$ and $0.1 \%$ strain. These strains are applied in isostatic compression and in isobaric conditions with the following conditions: uniaxial compression, uniaxial and biaxial tractions, and pure shear. These are obtained by a change in the dimensions of the box or its shape (pure shear). From each simulation, after stabilization of the stress, a component of the elastic tensor is deduced. Some simulations are redundant to verify the quality of predictions.

The bulk melt is obtained by melting a crystal composed of 9600 atoms with a free surface at high temperature $(2000 \mathrm{~K})$. The crystal melts progressively from the free surface. After complete melting, the size of the box is modified to reduce the pressure to zero for each simulation temperature. The depression experiments are done

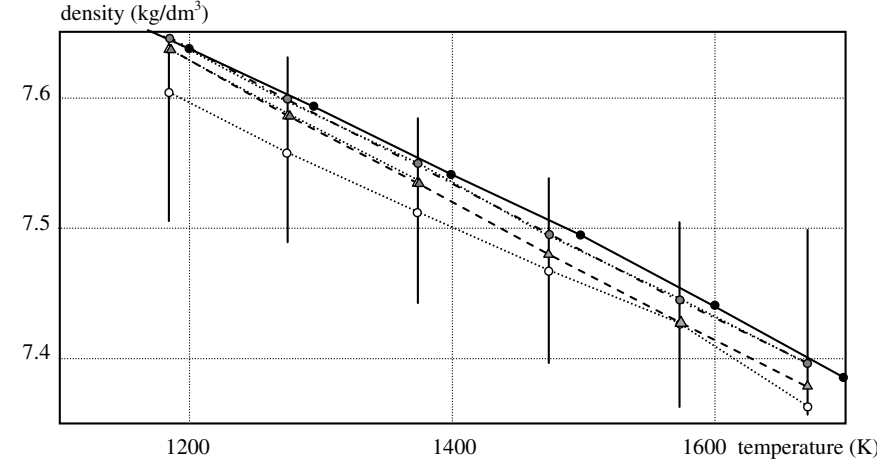

Fig. 1. Simulated density $\bullet$ as a function of temperature and experimental data: $\triangle$ Basinski [12], $\bigcirc$ Cockett [13], $\triangle$ Kohlhaas [14], O Lucas [15].

by a progressive expansion of the box in one direction.

The 96000 atom system in a semi-solid system is balanced at the equilibrium melting temperature $(1710 \mathrm{~K})$ found during our accuracy test of Kojima-Rosato's force-field. The atoms at the boundary layer encountered by periodicity of the crystal perpendicular to the thickness are fixed in position to avoid the effect of the crystal thickness on the depression of the melting temperature, i.e. Gibbs-Thomson's law. The lateral dimensions of the box are fixed at the values deduced from simulations done on the crystal at the same temperature. In this periodic model the increase in the box length, along the normal of the growth front, i.e. the dense plane [111], induces a depression in the solid phase and in the melt.

\section{Results and discussion}

\subsection{Elastic deformation of a fcc iron crystal}

The density evolution of the fcc crystal is deduced from molecular dynamics simulation from $300 \mathrm{~K}$ up to $1700 \mathrm{~K}$ without mechanical constraint. It is possible to partly validate the force-field by comparison of simulated with experimental values of the density (Fig. 1).

The maximum deviation of simulated density from experiments is less than $5 \%$. Uniaxial traction is applied under isothermal conditions. The lateral dimensions of the crystal are modified to analyze their influence on the components of the stress tensor and to measure Poisson's ratio (Fig. 2a). 


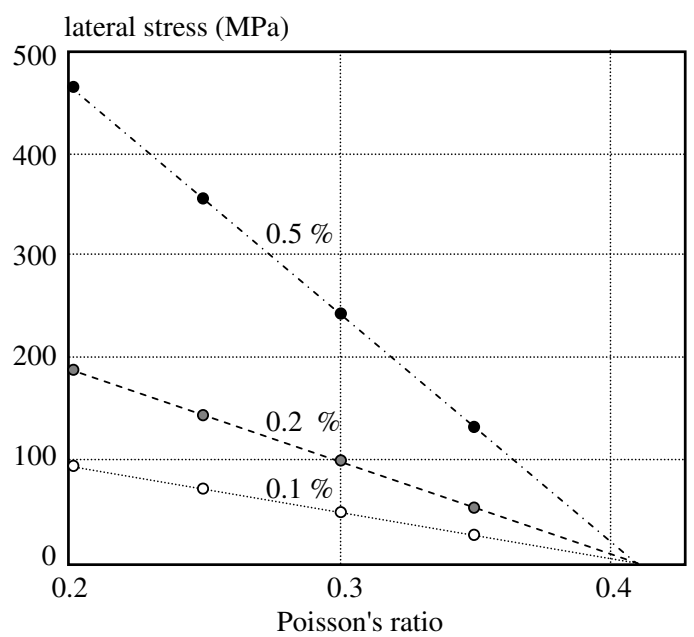

(a)

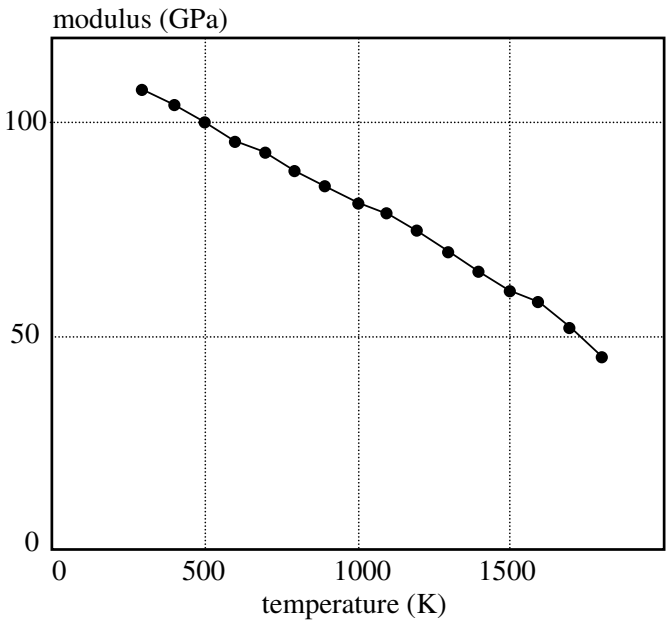

(b)

Fig. 2. (a) Prediction of the Poisson coefficient along the $\langle 010\rangle$ direction; (b) thermal evolution of the elastic modulus along the $\langle 010\rangle$ direction.

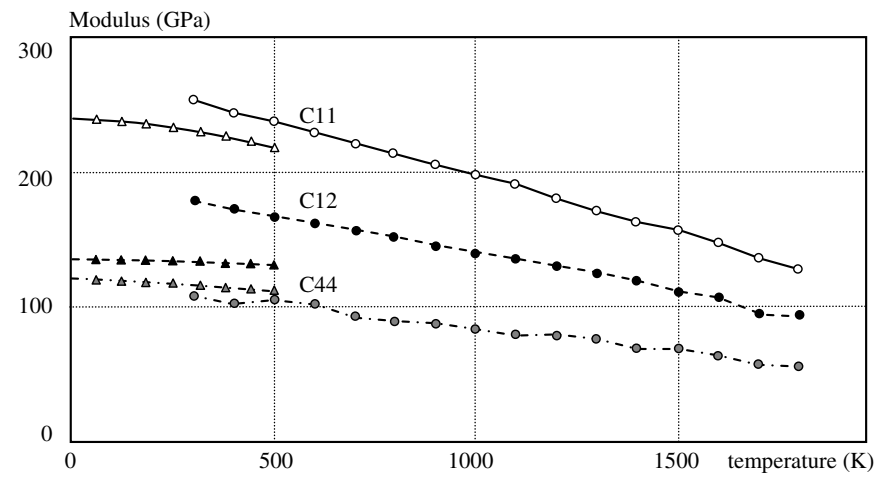

Fig. 3. Thermal evolution of the elastic tensor $\bigcirc$ and comparison with experimental data obtained at low temperature on an iron monocrystal $\Delta[16]$.

The reduction of the lateral stress is able to predict the elastic modulus in plane stress conditions (Fig. $2 b$ ).

Poisson's ratio for the $\langle 100\rangle$ crystallographic direction deduced from simulation is 0.41 . This value is slightly higher than the experimental value, $0.365-0.385[13,14]$. The elastic modulus at $300 \mathrm{~K}(E=108 \mathrm{GPa})$ is very low compared with experimental data on a polycrystalline iron $(E \sim 200 \mathrm{GPa})$ at this temperature. This value strongly depends on the crystallographic orientation, from $105 \mathrm{GPa}$ to $270 \mathrm{GPa}$ [15]. The thermal dependence of the elastic modulus is very weak. At $1700 \mathrm{~K}$ the elastic value is about half the value at $300 \mathrm{~K}$. Strong mechanical properties are predicted up to the melting temperature and should be confirmed by the analysis of the elastic tensor as a function of temperature.

We use the facility implemented by DL_POLY to obtain the temperaturedependent elastic constants from long-run MD simulations. The isobaric experiments under uniaxial traction, compression and biaxial traction give redundant data on the C11 and C12 components of elastic tensor, and pure shear simulations under isobaric conditions provide values for the $\mathrm{C} 44 \mathrm{com}-$ ponents. These three components of the fcc elastic tensor are predicted from $300 \mathrm{~K}$ up to $1800 \mathrm{~K}$ (Fig. 3).

We are not able to compare the values with experimental values on fcc crystal but only with values measured on bcc pure iron monocrystal ( $\alpha$ phase) [16]. They are very similar in the common temperature range. Thus, the main difference appears in C12, which is higher in simulation than deduced from experiments. This discrepancy can result from the difference in crystalline phases; our results are very similar to the predictions made by ab-initio simulations [17]. Cauchy's criterion C12 = C44 is satisfied neither in the experiments nor in the simulation. This is usual in transition metals. The weak decrease in the elastic modulus and in the components of the elastic tensor with 
temperature implies that the crystals should be stiff and resistant to cracking in the hot tear process. It can be noted that a perfect crystal is modeled by molecular dynamics with no dislocation to initiate plastic deformation. The weakness of the mushy zone should be found in other zones of the mushy zone, in the melt or the melt-crystal interface to explain the initiation process.

\subsection{Cavitation and properties in the melt}

A balanced melt is initiated following the procedure described in Chapter 2. The simulations are performed at different temperatures from $2000 \mathrm{~K}$ to $1600 \mathrm{~K}$. At each temperature, the size of the box is increased by $0.05 \mathrm{~nm}$ steps for $20 \mathrm{ps}$. The density decreases and a depression gradually develops inside the melt. At the end of each step the pressure stabilizes to an equilibrium value. The pressure and the properties are measured during the last 5 ps of each step (Fig. 5a). At a critical pressure a void spontaneously appears and a spherical void expands very quickly. This is a homogeneous nucleation process for the void formation when heterogeneous nucleation is expected in experiments. The effect of this specific process will be discussed later. The size of the void increases and stabilizes to a nanometric size (Fig. 4). During this period the depression is reduced and then is stabilized to a constant negative pressure after a few oscillations. This phenomenon is the same whatever the temperature in the domain of interest.

An example of the cavitation process is illustrated in Figure 4.

The bubble is almost spherical once a stable state is reached. There is no gas inside the void, while it should appear during experiments. Some complementary simulations are done with a larger number of atoms and then a larger simulation box, leading to almost the same results. The final size of the bubble increases with the size of the molecular dynamics simulation. The final size reaches $0.9 \mathrm{~nm}$ for the smaller box and $1.4 \mathrm{~nm}$ for the larger one. The pressure is measured during the whole process of expansion of the

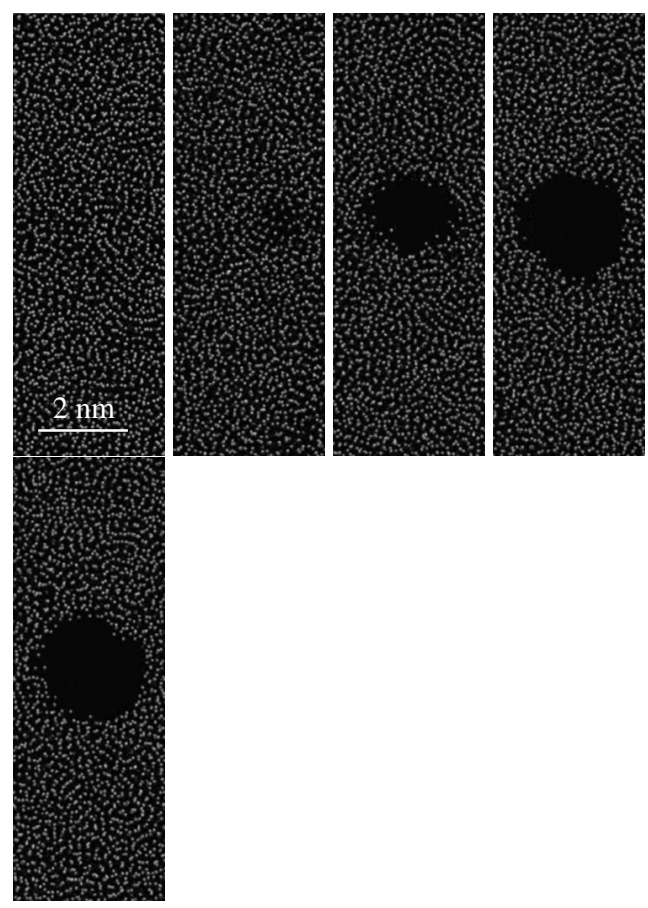

Fig. 4. Cavitation process in a thin cut of the 3D simulation domain of melt. $T=2000 \mathrm{~K}$, $\Delta t=1$ ps between two consecutive images.

Table 1. Critical pressure for cavitation as a function of the temperature of the melt.

\begin{tabular}{cccccc}
\hline Temperature (K) & 1600 & 1700 & 1800 & 1900 & 2000 \\
\hline Pressure for cavitation $(\mathrm{GPa})$ & 4.5 & 4.8 & 4.1 & 3.9 & 3.5 \\
\hline
\end{tabular}

box size, cavitation initiation, expansion and stabilization (Figs. 5a, 5b).

The pressure decreases during the expansion of the box to a critical value (Pc) where a cavitation appears. During the expansion of the cavity the pressure increases and then stabilizes (Fig. 5a). The present evolution for pure iron slightly depends on temperature with some deviations observed at low temperature during the first steps of depression (Fig. 5b). When a higher temperature of the melt is considered the curves are shifted to larger volumes as an effect of the thermal expansion coefficient of the melt (Fig. 5b). A higher critical pressure $P C$ results from a decrease in the temperature (Tab. 1).

The initiation of cavitation requires higher depression at lower temperature. These values are highly negative and are similar to previous MD results obtained on aluminum melt $P c=-3.2 \mathrm{GPa}$ [5]. The nucleation theory of cavitation is able to predict 


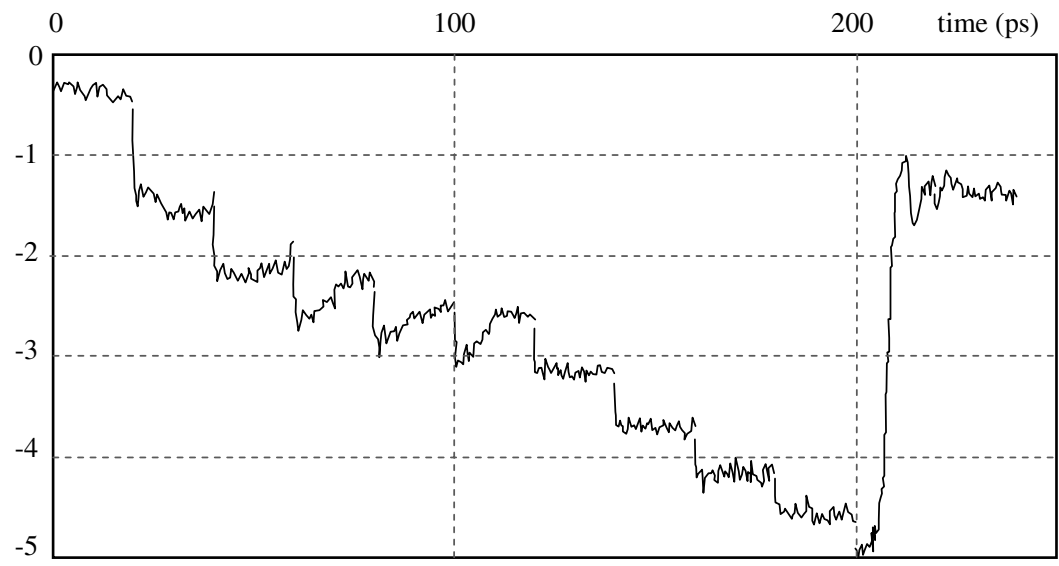

pressure $(\mathrm{GPa})$

(a)

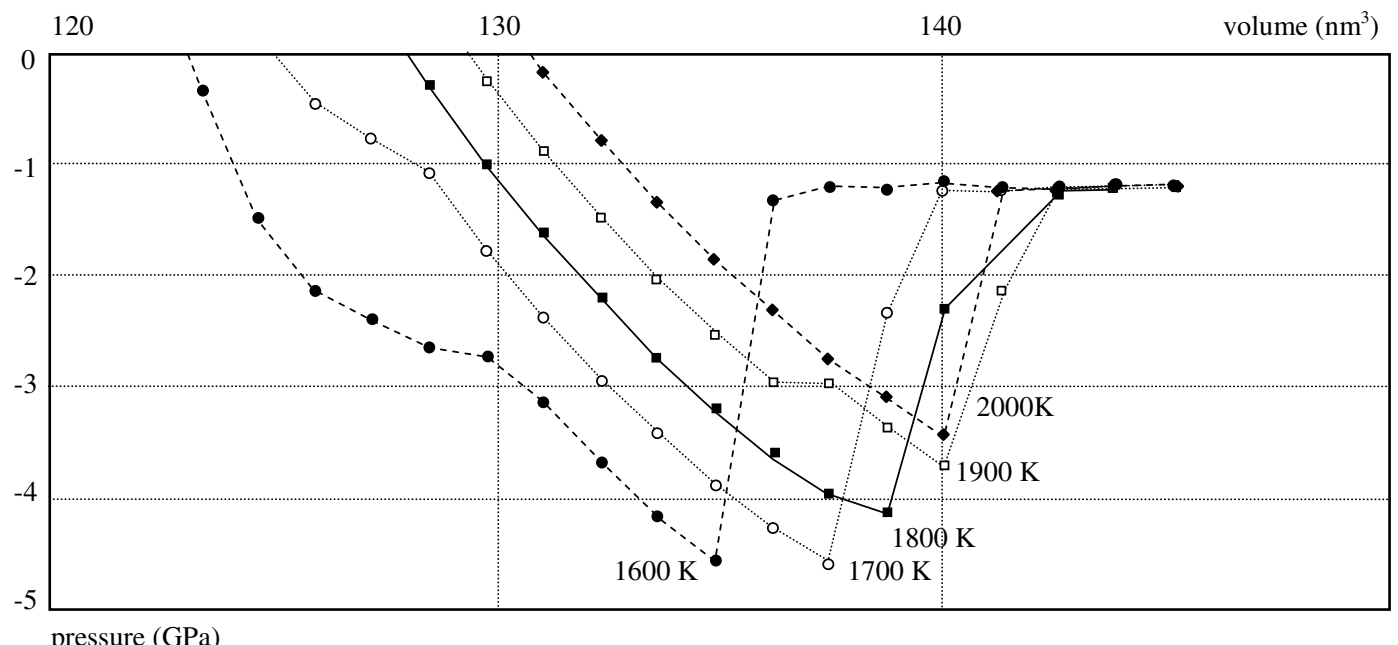

(b)

Fig. 5. (a) Pressure evolution during the box size expansion up to cavitation $(1600 \mathrm{~K})$; (b) effect of temperature on the stabilized pressures during the box expansion and cavitation.

this critical pressure as a function of the work necessary to reach a critical radius $P_{c}[18]$ :

$$
\begin{aligned}
W^{*} & =\frac{16 \pi \gamma^{3}}{3 p^{2}} \\
P c & =\left[\frac{16 \pi}{3 k_{b} T} \frac{\gamma^{3}}{\ln \left(N k_{b} T / h\right)}\right]^{1 / 2}
\end{aligned}
$$

where $P c$ strongly depends on the surface energy $\gamma$ and more slightly on the temperature T. $k_{b}$ and $h$ are the Boltzmann and Planck constants, respectively. The thermal dependence of $P c$ indirectly appears in Equation (2) through the thermal dependence of $\gamma$. Usually $P_{C}$ is defined from values of surface energies measured at a temperature at al- most atmospheric pressure, using Laplace's law [19]:

$$
\gamma=\frac{R P}{2}
$$

where $R$ and $P$ are the radius of the void and the applied pressure, respectively. The critical pressure is $-7.08 \mathrm{GPa}$ with a surface energy $\gamma=1.85 \mathrm{~N} / \mathrm{m}$ for an iron melt at $1800 \mathrm{~K}$, near-atmospheric pressure [19]. This value and the critical depression predicted by molecular dynamics are in the same depression range (Tab. 1, Fig. 5b). This value is very high for a pure iron melt compared with predictions resulting from modeling of hot tearing $[1,6]$. 
The surface energy $\gamma$ is the main parameter of Equation (2). It is possible to predict its value from the measurements of the equilibrium radius of the void and the associated pressure in equilibrium conditions. These values can be deduced from the equilibrium state of the void after its expansion (Figs. 5a, 5b). The application of Equation (3) leads to a value $\gamma=0.88 \pm 0.01 \mathrm{~N} / \mathrm{m}$ from $1600 \mathrm{~K}$ to $2000 \mathrm{~K}$ (Fig. 6).

Therefore, the surface energy $\gamma$ can be considered as almost constant. The value is about half of the surface energy measured from experiments. The experimental measurements are made with a very small depression. The melt is weakly perturbed from its equilibrium state at atmospheric pressure. The molecular dynamics simulations predict only small voids inside a larger box. This size implies a very large depression in the melt for the equilibrium state. Atoms in this equilibrium state have a density which is significantly lowered from experimental conditions. The atoms which move at the void surface and inside the melt are less constrained under depression than nearatmospheric pressure. The surface energy deduced from molecular dynamics simulations should be lower than the experimental value. The application of this surface energy $(\gamma=0.88 \mathrm{~N} / \mathrm{m})$ in Equation (2) leads to a critical pressure $P_{c}=-2.35 \mathrm{GPa}$. The critical pressure is included inside the range predicted by the model with the two possible values of the surface energy. The surface energy deduced from molecular dynamics simulation is in reasonable agreement with the expected value deduced from experiments.

This higher mobility of atoms under depression should also act on the viscosity in a similar way to temperature. The physical origin is different: the thermal effect comes from the change in the kinetic energy, while the depression effect comes from the potential energy. A larger depression results from the expansion of the box size. The mean distance between neighbor atoms must increase. The attractive part of the force field is greater than its repulsive contribution. The viscosity is deduced from the diffusion coefficient from Einstein's equation on Brownian motion [20]. The diffusion

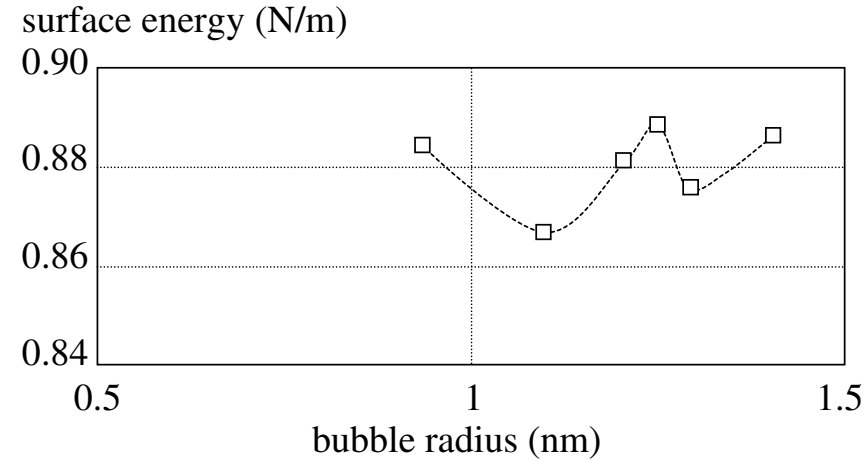

Fig. 6. Surface energy as a function of void radius.

coefficient $D$ is deduced from molecular dynamics simulations with two different methods: the analysis of the mean square displacement of atoms as a function of time, and the autocorrelation function of the atom speed, using the Green-Kubo formula. The diffusion coefficient is measured during the depression steps up to the cavitation event. At each step the two methods are applied as a function of time to predict the diffusion coefficient (Fig. 7a) and then the viscosity (Fig. 7b).

The experimental values of the diffusion coefficient under atmospheric pressure, $D=4.16 \times 10^{-9} \mathrm{~m}^{2} / \mathrm{s}(1833 \mathrm{~K})$ and $D=$ $4.91 \times 10^{-9} \mathrm{~m}^{2} / \mathrm{s}(1900 \mathrm{~K})$ [21], are almost identical to the prediction of our model. The validation of the method is also applied to the viscosity measurement as a function of temperature under atmospheric pressure (Fig. $7 b$ ). The viscosity predicted by molecular dynamics simulations varies from $1.5 \mathrm{mPa} . \mathrm{s}(2000 \mathrm{~K})$ to $3.25 \mathrm{mPa} . \mathrm{s}(1600 \mathrm{~K})$ (Fig. 7b). It is possible now to consider the effect of pressure on the melt viscosity (Fig. 7c). The depression induces a decrease in the viscosity, as expected. The piezo-dependence of the viscosity increases when the temperature decreases (Fig. 5c). The effect can affect the feeding of the mushy zone. It is a coupled effect between the thermal effect and the diffusion through the network of porosity.

The expansion of the void can also be analyzed from these molecular dynamics simulations of iron melt. A short time, $4.1 \mathrm{ps,}$ is sufficient to expand the void to the final radius $R=1.4 \mathrm{~nm}$. It is possible to calculate the mean speed of the radius 


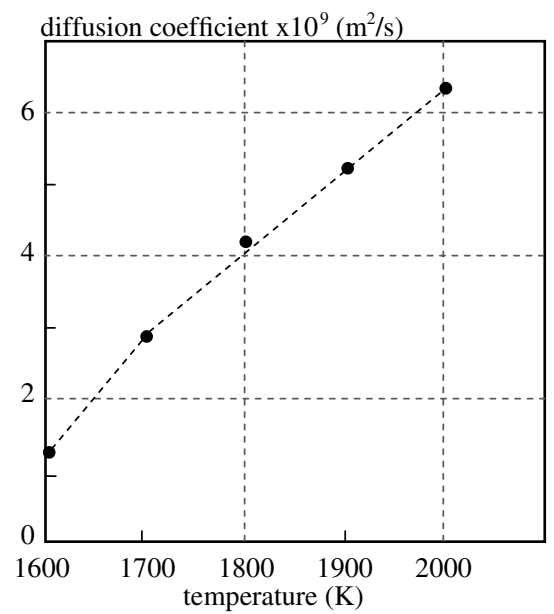

(a)

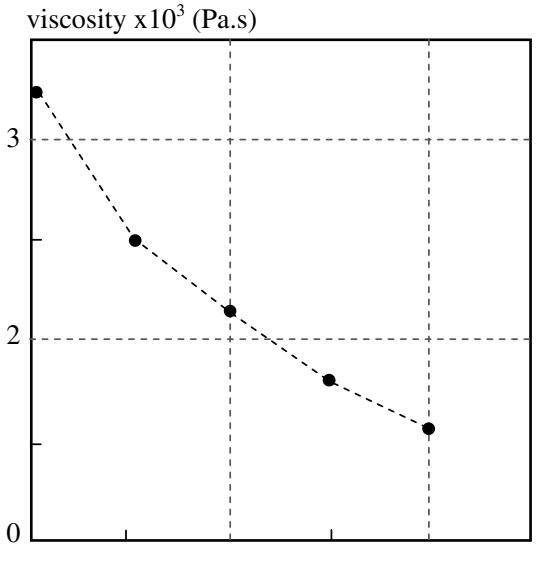

(b)

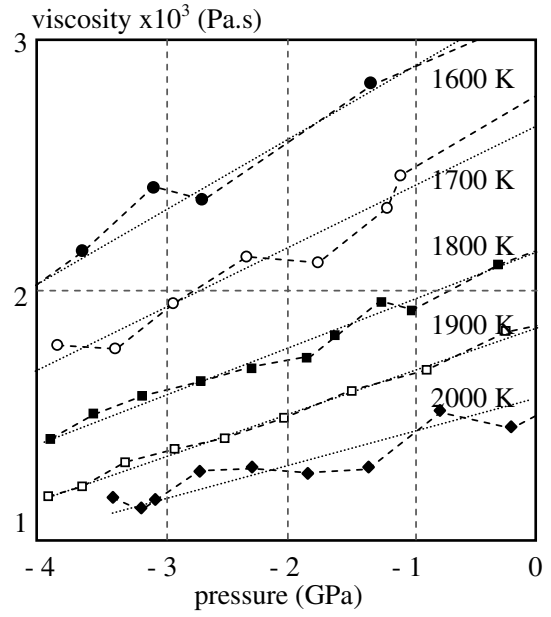

(c)

Fig. 7. (a) Thermal dependence of the diffusion coefficient modeled by molecular dynamics under atmospheric pressure; (b) thermal dependence of the viscosity at atmospheric pressure; (c) temperature and negative pressure effects on viscosity.

expansion: $V=340 \mathrm{~m} / \mathrm{s}$. It is associated with a pressure variation of $3 \mathrm{GPa}$ and a compression speed of $7.3 \times 10^{11} \mathrm{GPa} / \mathrm{s}$, assuming a mean initiation pressure of $P c=4.3 \mathrm{GPa}$ and $1.3 \mathrm{GPa}$ the final pressure in the melt near the void. This growth rate of the void is consistent with a similar molecular dynamics simulation of aluminum ( $G=400 \mathrm{~m} / \mathrm{s}$ ) [5]. This phenomenon is an undersonic compression with a very high speed variation of pressure. It should develop a compression wave in the melt during the expansion of the void.

\subsection{Cavitation and properties in the semi-solid state}

The cavitation appears inside a confined region of the mushy zone. It is necessary to explore the effect of the narrow proximity of the crystalline growth front on the cavitation process. The reference is made by the cavitation inside a pure melt (Sect. 2.2). The analysis of the effect of the crystal interface on cavitation requires a stable growth front with no crystallization or melting, i.e. the equilibrium melting temperature predicted from this force-field $T=1710 \mathrm{~K}$. The procedure applied to the melt (Sect. 2.2) is reproduced on the semi-solid domain in the vicinity of the crystalline growth front [111]. Contrary to Hoyt [5], who uses only the stress created by the solidification to create a bubble, in this work, after equilibration, the system volume is changed step by step to create a negative pressure inside the molten and crystalline phases. The expansion of the box along the normal of the crystalline surface induces a progressive depression and then a cavitation process in the melt (Fig. 8). No cavitation is observed inside the crystal.

These initiation processes observed inside the semi-solid domain and in the melt are different. Multiple void nuclei are initiated in the semi-solid domain (Fig. 8, 73 ps). Most of them quickly disappear $(\Delta t<1 \mathrm{ps})$ to produce only a single void (Fig. 8, 75 ps). It appears in the semi-solid system at a distance from the crystal surface with no specific location at nanometric distances. In the melt only one void appears during each simulation (Fig. 4).

Shorter periods of the equilibrium steps (2 ps) are chosen to follow the pressure evolution more precisely with smaller length increments of the box. The multiple nucleation formation of voids is not modified by the period of equilibration, i.e. the expansion rate of the box. The pressure as a function of the expansion of the semi-solid system seems to be very similar to those observed in the melt up to the first steps of cavitation (Fig. 9).

The pressure decreases regularly to $P_{C}=$ $-3.2 \mathrm{GPa}$ where the multiple voids appear. Then, the pressure suddenly decreases to a pressure $P=-4.4 \mathrm{GPa}$ where only one void remains. This pressure reached by one void 


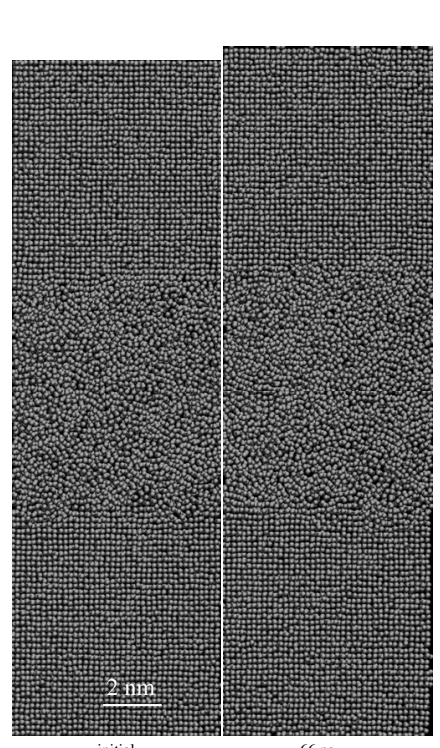

initial

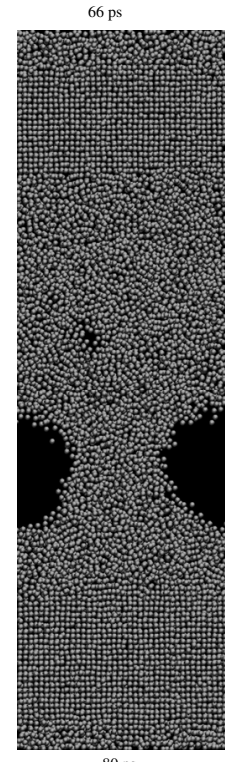

$80 \mathrm{ps}$

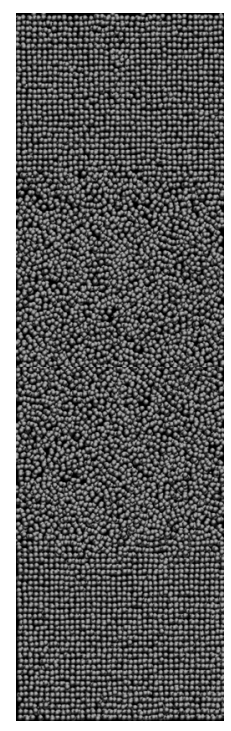

$68 \mathrm{ps}$

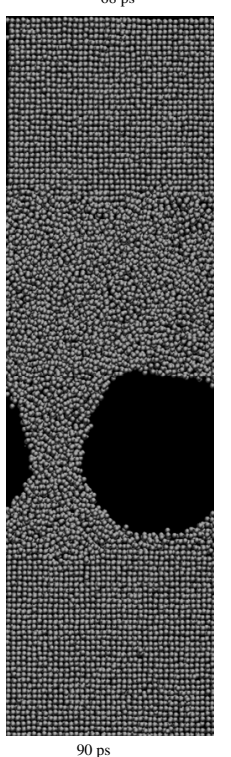

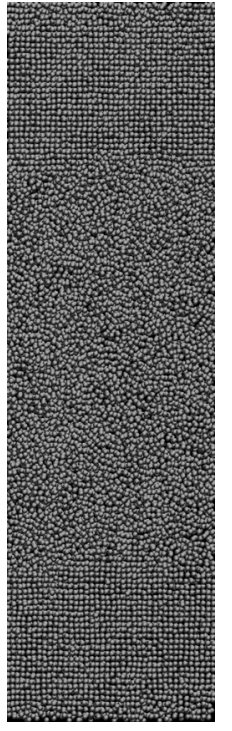

$70 \mathrm{ps}$

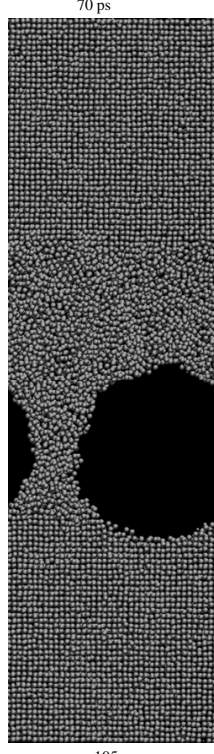

$105 \mathrm{ps}$

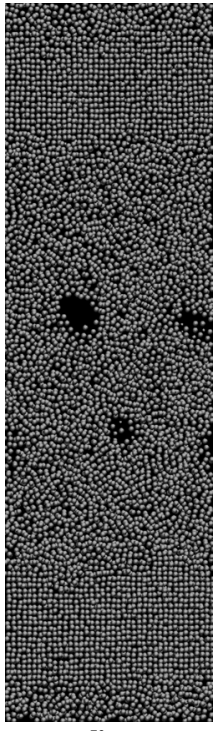

$73 \mathrm{ps}$

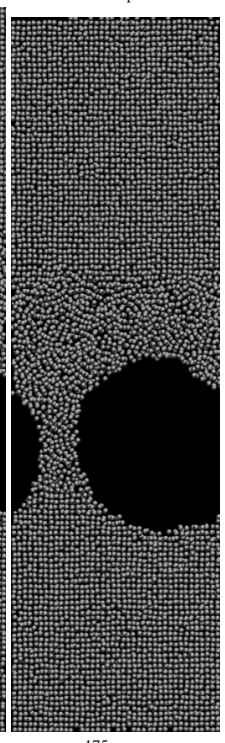

$175 \mathrm{ps}$

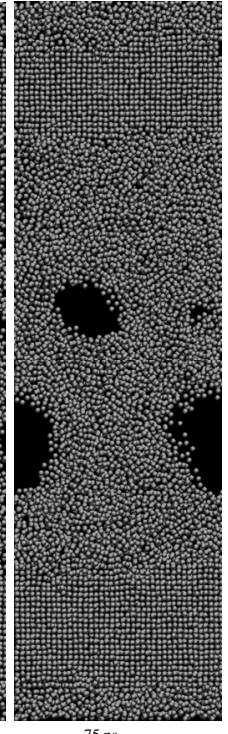

$75 \mathrm{ps}$

Fig. 8. Evolution of the semi-solid domain and cavitation inside the melt as a function of time.

in the melt and in the semi-solid state are almost the same (-4.4 GPa). It is the first step for the expansion of the void. The multiple void nucleation at an "effective" cavitation pressure $P_{c}=-3.2 \mathrm{GPa}$ is necessary to promote this single initial void. The creation of multiple voids leads to the critical pressure of cavitation. When the multiple cavities appear they are spontaneously converted to a single void with a pressure decrease. No reverse process seems possible at this stage. The formation of multiple voids is the critical step.

Following the hypothesis that multiple nucleation of voids near the crystal surface is the critical step, the nucleation of voids should appear near the crystal surface for a critical pressure $P c=-3.2 \mathrm{GPa}$. This heterogeneous nucleation process seems to be favored by the local organization in the melt near the crystal surface. Hoyt et al. concluded that the interface does not affect the void nucleation [5]. Our observations lead to a preferential nucleation of the voids near the crystal surface.

The initial expansion of the void has almost the radial expansion rate measured in the melt $\sim 340 \mathrm{~m} / \mathrm{s}$. This expansion speed evolves to an anisotropic speed progressively reduced during the growth of the 


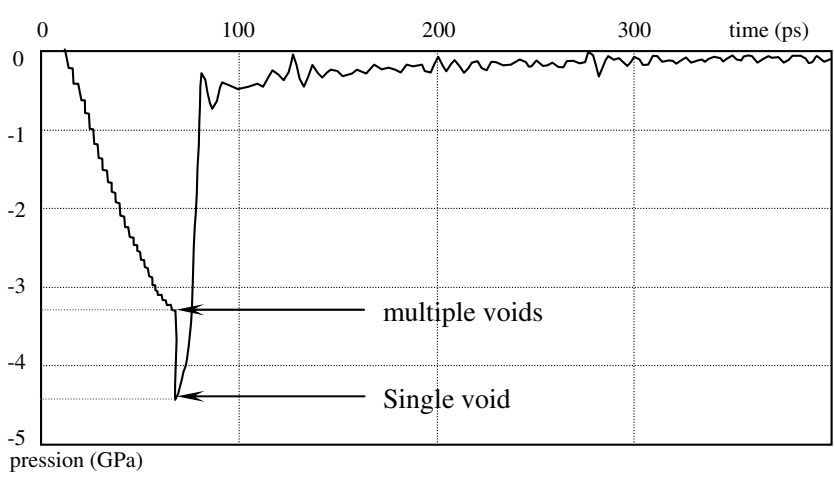

Fig. 9. Evolution of the pressure during depression and cavitation in a semi-solid iron system.

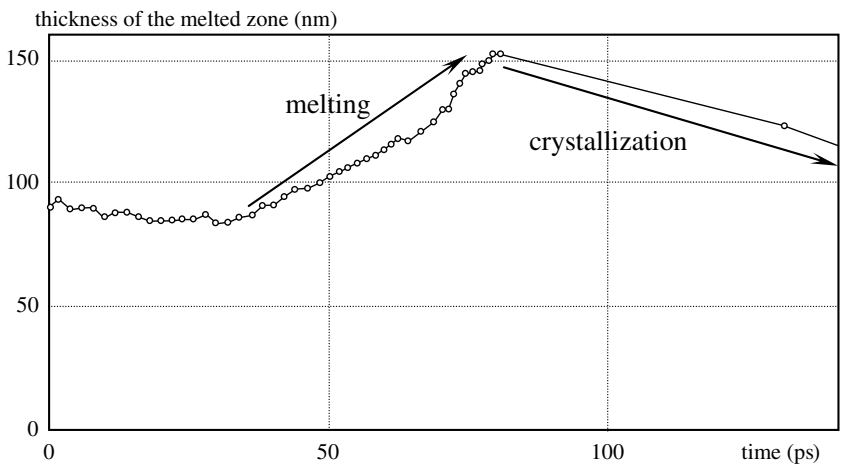

Fig. 10. Evolution of the melt layer between two crystal interfaces as a function of time during the expansion of the volume to apply a depression.

void, leading to an anisotropic shape of the void in contact with the crystal (Fig. 10). This mechanism is more complex than in the melt. It is coupled with the evolution of the crystal growth front (Fig. 10).

A more detailed analysis of the evolution of the position of this crystal surface is necessary to explain the coupling with the void formation. This is not a strict interface. The probability density function of the presence of an atom at a defined position along the thickness progressively decreases along 8 crystal layers. By convention, we define the crystal interface by the position where the density probability is half of the value in the inner crystal. The position of this interface is followed during depression up to the end of the cavitation process. The easiest way is to follow the thickness of the melted zone (Fig. 10).

During the first period down to about $-1 \mathrm{GPa}$ the crystalline interface is stable. Then, the crystal progressively melts, i.e. the molten layer grows (Fig. 10). The melting rate increases with the depression. The analytical form of the rate dependence on depression is difficult to define. Thus, the mean melting speed of the crystal in this depression range is $G \sim 70 \mathrm{~m} / \mathrm{s}$. This growth rate is very high. During the determination of the equilibrium melting temperature $T=$ $1710 \mathrm{~K}, \mathrm{G}=0 \mathrm{~m} / \mathrm{s}$ we checked the thermal dependence of the growth rate $G$. A temperature variation of $100 \mathrm{~K}$ from the equilibrium temperature provokes only $G=0.7 \mathrm{~m} / \mathrm{s}$ of the growth front. This thermal effect is two orders of magnitude lower than the pressure effect observed during these simulations. If a cavity is closed and the depression acts for $1 \mathrm{~ms}$, the pressure effect predicts a melting of about 70 microns of the dendrite structure. This size reduction is able to open a closed cavity. The prediction of the exact behavior requires a complete coupled model which is outside the scope of the present paper. This phenomenon develops up to the initiation of the voids. When the void appears and grows, the depression is lowered and there is a competition between void expansion and recrystallization. In the simulation the crystalline front grows up to a position which corresponds to a larger extent to the initial one when the pressure was reduced to 0 bar in isothermal conditions. This is due to the existence of the void. The final pressure is slightly negative, $-0.1 \mathrm{GPa}$, when the equilibrium value is $-1.3 \mathrm{GPa}$ in the melt.

\section{Conclusion}

These series of molecular dynamics simulations were done in very simple conditions which cannot be decoupled in experiments. The combination of their results leads to a scheme for the initiation of hot tear defects. The model predicts very high mechanical properties of the crystal in the range of melting temperatures. We conclude that the decrease in the mechanical properties is not the main origin of the hot tearing phenomenon. Our model describes the behavior of a fcc iron crystal with no dislocation. Dislocations would play a role in such a process but the perfect crystal has half of the ambient mechanical properties. The crystal seems too stiff to promote hot tearing. 
During crystallization inside a closed cavity the depression promotes a partial melting of the crystals. There is competition between cooling and depression on the solidification process. The magnitude of the piezo-dependence of the melting rate is able to counterbalance the cooling effect. The crystalline lamella can be thinned during the step of depression inside a confined molten zone. These voids should appear near the crystalline surface and induce a pressure wave which can break the thinned crystals due to their partial remelting. During the expansion of the void, the crystal recrystallizes with the void and possibly creates cracks inside. It must be noticed that the void size in the cavities must be larger than the ones simulated by molecular dynamics. The final void size in molecular dynamics is a consequence of the size of the domain simulated. Molecular dynamics is able to predict only the initiation of the voids and the first steps of their expansion. The real size expansion is outside the scope of this type of simulation. One experimental way to check this hypothesis may be to analyze possible tiny crystalline variations, due to melting and recrystallization processes, in a border of a final void. The time predicted for the void formation process is too short to expect an in situ observation. One must also point out the large difference between the value of the critical pressure predicted by molecular dynamics simulations and hot tearing models. The origin of this discrepancy should be studied in more detail.

Another scheme could also consider that the remelting is sufficient to reopen some closed cavities. At this point, a feeding of the cavity is possible with a melt viscosity which depends on the temperature, but also on depression. A modeling of the complete process should consider the depression effect. A large depression should reduce the viscosity and then favor the feeding of the cavity. However, this process cannot promote crack initiation.

\section{References}

[1] J. Campbell, Castings, ButterworthHeinemann, Oxford, United-Kingdom, 1991

[2] M. Rappaz, J.M. Drezet, M. Gremaud, Metall. Mater. Trans. A 30A (1999) 449-55

[3] J.A. Spittle, A.A. Cushway, Met. Technol. 10 (1983) 6-13

[4] J.M. Drezet, J. Phys. IV 9 (1999) 53-62

[5] S. Vernede, J.A. Dantzig, M. Rappaz, Acta Mater. 57 (2009) 1554-1569

[6] J.J. Hoyt, A.A. Potter, Metall. Mater. Trans. A 43A (2012) 3972-3977

[7] V.N. Mineev, A.I. Funtikov, Phys. Usp. 47 (2004) 671-686

[8] Y.D. Fomin, V.N. Ryzhov, V.V. Brazhkin, arXiv: 1301.7158 (2013)

[9] V. Rosato, M. Guillopé, B. Legrand, Phil. Mag. A 59 (1989) 321-336

[10] R. Kojima, M. Susa, Sci. Techn. Adv. Mater. 5 (2004) 497-502

[11] F. Ducastelle, F. Cyrot-Lackmann, J. Phys. Chem. Sol. 31 (1970) 1295-1306

[12] Z.S. Basinski, R.W. Hume, A.L. Sutton, F.R.S Sutton, Proc. Roy. Soc. London A 229 (1955) 459-467

[13] G.H. Cockett, C.D. Davis, J. Iron steel Inst. (London) 201 (1963) 110-115

[14] R. Kohlhaas, P. Dunner, N. SchmitzSpranghe, Z. Angew. Phys. 23 (1967) 245-249

[15] L. Lucas, Mém. Sci. Rev. Mét. 69 (1972) 763772

[16] S. Watanabe, Y. Tsu, K. Takano, Y. Shiraishi, Jpn. Inst. Met. 45 (1981) 242-249

[17] G.Y. Guo, H.H. Wang, Chin. J. Phys. 38 (2000) 949-961

[18] J.C. Fisher, J.H. Hollomon, D. Turnbull, J. Appl. Phys. 19 (1948) 775-784

[19] J. Campbell, J. Appl. Phys. D 1 (1968) 10851088

[20] A. Einstein, Annal. Phys. 17 (1905) 549-560

[21] T. Lida, R.I.L. Guthrie, The physical properties of liquid metals, Clarendon Press, Oxford, 1988 